\title{
The Early Trauma Inventory Self Report-Short Form: Psychometric Properties of the Korean Version
}

\author{
Ju-Ri Jeon', Eun-Ho Lee', Sun-Woo Lee ${ }^{2}$, Eu-gene Jeong ${ }^{1}$, \\ Ji-Hae Kim¹, Dongsoo Lee ${ }^{1,2}$ and Hong Jin Jeon ${ }^{1 凶}$ \\ ${ }^{1}$ Department of Psychiatry, Depression Center, Samsung Medical Center, Sungkyunkwan University School of Medicine, Seoul, Republic of Korea \\ ${ }^{2}$ Social Mental Health Institute of Samsung Medical Center, Seoul, Republic of Korea
}

Objective Experiencing traumatic events in childhood is related to various psychiatric problems in adulthood, and a comprehensive tool for measuring childhood trauma is necessary in this field. This study aimed to examine the psychometric properties, and factor structure of the Korean version of the Early Trauma Inventory Self Report-Short Form (ETISR-SF). ETISR-SF measures the childhood trauma, including physical, and emotional sexual abuse, as well as general traumas.

Methods A clinical and nonclinical samples comprising of 97 subjects from a local community, and 207 patients with the ETISR-SF, were assessed. Other tools, including the Childhood Trauma Questionnaire-Short Form (CTQ-SF), the Beck Depression Inventory (BDI), and the Beck Anxiety Inventory (BAI) were used to assess clinical symptoms. Additional data from 69 college students was used to examine the test-retest reliability.

Results The original four-factor model was supported by the confirmatory factor analysis scale $\left[\chi^{2}(351, n=304)=3374.025, p<0.001\right.$, $\mathrm{TLI}=0.969, \mathrm{CFI}=0.972, \mathrm{RMSEA}=0.030$ ]. The ETISR-SF was found to be a reliable instrument (Cronbach's $\alpha=0.869$ ). Comparison of the ETISR-SF scores discriminated the clinical group from that of the control group. The measure showed good convergent and divergent validity, in that the scores were correlated higher with the scores on the CTQ-SF (0.691) than with the scores on the BDI or BAI (0.424, 0.397 respectively). The ETISR-SF was found to be temporally stable, showing the moderate to high correlation (0.844).

Conclusion These findings suggest that the Korean version of the ETISR-SF appears to be a reliable and valid instrument for the measurement of reported childhood trauma.

Psychiatry Investig 2012;9:229-235

Key Words Early trauma, Trauma inventory, Validation study, Depression.

\section{INTRODUCTION}

Childhood Trauma is defined as an adverse early life experience, including physical, emotional, sexual abuse and general traumatic experience. ${ }^{1}$ Experiencing childhood trauma is associated with difficulties, such as depression, anxiety, substance abuse, and even suicide, in adolescence, young adulthood or later life. ${ }^{2}$ Early studies on childhood trauma focused on sexual abuse and its consequences, which include interpersonal conflicts or Borderline Personality Disorder. ${ }^{3}$ Yet,

Received: January 19, 2012 Revised: May 2, 2012

Accepted: May 16, 2012 Available online: September 6, 2012

$\triangle$ Correspondence: Hong Jin Jeon, MD, PhD

Department of Psychiatry, Depression Center, Samsung Medical Center, Sungkyunkwan University School of Medicine, 50 Irwon-dong, Gangnam-gu, Seoul 135-710, Republic of Korea

Tel: +82-2-3410-3586, Fax: +82-2-3410-0050, E-mail: jeonhj@skku.edu

(c) This is an Open Access article distributed under the terms of the Creative Commons Attribution Non-Commercial License (http://creativecommons.org/licenses/by$\mathrm{nc} / 3.0$ ) which permits unrestricted non-commercial use, distribution, and reproduction in any medium, provided the original work is properly cited. other forms of abuse are also important in understanding the mental health. Physical abuse has effect on many areas from psychological adjustment in everyday life to suicidal behaviors. ${ }^{4}$ In a recent study, emotional neglect was also related to suicidal ideation and suicidal behaviors. ${ }^{5}$ In addition to abuse, general trauma, such as natural disaster or family bereavement, can also be a cause of posttraumatic stress disorder (PTSD) or depression. ${ }^{6}$ In spite of the significance of trauma, recalling traumatic events without specific forms of questionnaires could limits the range of traumatic events. ${ }^{7}$ For this reason, a structured and comprehensive tool for measuring traumatic events is needed to understand patients' symptoms better. $^{8}$

There are a number of instruments that assess the childhood trauma, with each of these measuring somewhat different dimensions with a wide range of psychometric properties. Some are clinician-administered assessments, while others are self-administered reports. ${ }^{9,10}$ Some focus on the limited 
dimensions of trauma, such as sexual abuse, whereas, others include a wide range of abuse, but not general trauma. ${ }^{11}$ As mentioned above, a wide range of traumatic events influence individual's clinical symptoms, and in this matter, a comprehensive measurement can be useful in this field.

The Early Trauma Inventory (ETI), one of the trauma measurements, is a semi-structured interview, which assesses the four domains of physical, emotional, sexual abuse, and general traumatic experience, and then addresses the most serious trauma in an additional question. ${ }^{1}$ For each item of the ETI, an interviewer has to assess the frequency of abuse/trauma by the developmental stage, the onset and termination of abuse/ trauma, and the perpetrator/cause of the abuse/trauma. The ETI is a comprehensive assessment tool that has good psychometric properties. However there are some limitations to the ETI: 1) It takes an hour, 2) responders could avoid reporting their experience face to face, and 3) trained staffs are needed to administer it. Due to these limitations, a self-report version of the ETI was developed, and then a shortened version of the ETI-SR was also introduced. ${ }^{12}$ The Early Trauma Inventory Self Report-Short Form (ETISR-SF) consists of 27 items in the four domains of physical, emotional, and sexual abuse, as well as general trauma. It categorically assesses the existence of these events before age 18 .

To date, there have been no studies validating this scale with the Korean samples. It is necessary to comprehensively assess the personal traumatic events in both the clinical setting and in research. Therefore, in the present study, we primarily examined the psychometric properties and factor structures of the Korean version of the ETISR-SF, using a community sample and a clinical sample.

\section{METHODS}

\section{Participants}

The participants consisted of 97 people without any psychiatric history, and 207 out-patients who visited the Depression Center. They were enrolled in the Depression Center Research Project, and have been interviewed by trained clinical psychologists. They completed a 30-minute self report. Additionally, the data from 69 college students were collected to examine the test-retest reliability.

The community sample consisted of 97 people (30 males, 67 females) who enrolled in the study through advertisements. The age of the participants was between 19 and 66 years $(M=44.05, S D=12.85)$. For males, the mean age was 41.50 ( $\mathrm{SD}=12.33)$, and for females, it was $42.30(\mathrm{SD}=13.15)$. Age difference by gender was not significant.

The clinical sample consisted of 207 patients (61 males, 146 females), who were recruited from the out-patients of the De- pression Center of the Samsung Medical Center in Seoul, Korea. Data from patients above age 18 were collected because the ETISR-SF assesses traumatic events that occurred before 18. The age of the participants was between 19 and 65 years $(\mathrm{M}=45.10, \mathrm{SD}=13.24)$. For males, the mean age was 41.43 $(\mathrm{SD}=14.76)$, and for females, the mean age was $46.64(\mathrm{SD}=$ 12.29). Age difference by gender was significant $(\mathrm{p}<0.05)$. Among the clinical sample, 121 (58.5\%) participants were primarily diagnosed with major depressive disorder, 17 (8.2\%) with depressive disorder not otherwise specified, and 6 (2.9\%) with dysthymic disorder. Twelve (5.7\%) participants had bipolar disorder, most recent episode depressed, and 33 $(16 \%)$ were diagnosed as having anxiety disorders. Seven (3.4\%) participants had adjustment disorder and the remainder included 4 patients with Undifferentiated Somatoform Disorder, 2 with alcohol problems and 1 with an eating disorder. The remaining 4 participants were only given secondary diagnoses, two of which were major depressive disorder, one bipolar disorder not otherwise specified, and the other bipolar II disorder. Secondary comorbid diagnoses included depressive (7\%), anxiety (13\%), alcohol (2\%), and eating (2\%) disorders. Among the additional data from 69 college students (21 males, 48 females), the age range of the students was between 19 and 29 years $(M=21.59, S D=0.464)$.

\section{Measures}

\section{The Early Trauma Inventory Self Report-Short Form ${ }^{12}$}

The ETISR-SF is a 27-item questionnaire, used for the assessment of physical, emotional, and sexual abuse, as well as general traumatic experience that may have occurred before the age 18. Each of the items is answered 'yes' (coded as 1) or 'no' (coded as 0 ). There are an additional three items, which are at the end of the questionnaire. One of these asks the subjects to choose the one event that had the greatest impact on his or her life, and the other two items measure the subsequent reactions, i.e. fear or depersonalization. The research team of a previous study initially translated the ETISR-SF into Korean by the original authors' permission, and through the back-translation, any ambiguous or mistranslated words were modified by a bilingual expert in the medical research area. ${ }^{5}$

\section{Childhood Trauma Questionnaire-Short Form ${ }^{13}$}

The CTQ-SF is a 28 -item retrospective self-report questionnaire with strong psychometric properties for community and treatment samples. It is widely used because it focuses on well-known childhood abuses and neglect. It assesses five dimensions of childhood maltreatment: physical abuse, emotional abuse, sexual abuse, physical neglect, and emotional neglect. Item response categories are scored from 1 to 5 (never 
true, rarely true, sometimes true, often true, very often true). We used a Korean version of the CTQ-SF. ${ }^{14}$ The internal consistency of the Korean version of the CTQ-SF was 0.79 and the test-retest reliability was 0.47 to 0.89 , among the sub-factors, which were a little bit lower than the test-retest reliability of the original version $(0.79-0.81) .^{13}$

\section{Beck Depression Inventory ${ }^{15}$}

The BDI is a 21 -item questionnaire designed to assess the degree of depressive symptoms present over a 1-week period. We used a Korean version of the BDI. ${ }^{16}$

\section{Beck Anxiety Inventory ${ }^{17}$}

The BAI is a 21-item instrument to measure the severity of anxiety symptoms. Each item is rated on a four-point Likerttype scale, ranging from 0 (not at all) to 3 (almost always). We used a Korean version of the BAI. ${ }^{18}$

\section{Procedure}

The ETISR-SF was administered to 97 healthy controls and 207 out-patients, all of whom gave informed consent. To examine the convergent and divergent validity of the ETISR-SF, all participants also completed the CTQ, BDI and BAI. Psychiatric diagnoses were based on the full version of Mini-International Neuropsychiatric Interview (MINI), for the purpose of establishing a Diagnostic and Statistical Manual of Mental Disorders $4^{\text {th }}$ edition (DSM-IV) diagnosis. ${ }^{19,20}$ The study was approved by the Institutional Review board of the Samsung Medical Center.

Additionally, the ETISR-SF was administered to 69 college students to examine the test-retest reliability. The students also gave their informed consent. The test-retest interval was three weeks.

\section{Statistical analyses}

Confirmatory factor analyses (CFA) were conducted with Mplus 6. We used the weighted least-square with the mean and variance (WLSMV) correction estimation method, which is appropriate for the binary data. To evaluate the goodnessof-fit of the CFA models, we selected multiple indices, including the Tucker-Lewis index (TLI), ${ }^{21}$ the comparative fit index (CFI), ${ }^{22}$ and the root mean square error of approximation (RMSEA). ${ }^{23}$ TLI and CFI values close to 0.95 indicate a good $\mathrm{fit}^{24}$ and RMSEA values of less than 0.05 indicate a good fit, with values between 0.05 and 0.08 indicating a reasonable fit. $^{25}$ To test the internal consistency, Cronbach's alphas were calculated, and in order to examine the group differences, $\mathrm{t}$ test was used. To examine the convergent and divergent validity of ETISR-SF, we calculated Pearson correlations with several measures, including CTQ-SF, BDI, and BAI. We used
SPSS 17.0 to analyze the descriptive statistics, internal consistency, test-retest reliability, and validity.

\section{RESULTS}

\section{Factor structure}

To test the stability of the factor structures of the ETISR-SF with a Korean sample, we conducted CFA on the whole data, including a community sample, to generalize the results. As a result, the goodness-of-fit indices for the Korean version of the ETISR-SF indicated that the data fitted well to the fourfactor structure model of the original scale $\left[\chi^{2}(351, n=\right.$ $304)=3374.025, \mathrm{p}<0.001, \mathrm{TLI}=0.969, \mathrm{CFI}=0.972, \mathrm{RMSEA}=$

Table 1. Factor loadings and squared multiple correlations from confirmatory factor analysis $(\mathrm{N}=304)$

\begin{tabular}{|c|c|c|c|c|}
\hline & $\begin{array}{c}\text { Factor 1: } \\
\text { general } \\
\text { trauma }\end{array}$ & $\begin{array}{c}\text { Factor 2: } \\
\text { physical } \\
\text { abuse }\end{array}$ & $\begin{array}{c}\text { Factor 3: } \\
\text { emotional } \\
\text { abuse }\end{array}$ & $\begin{array}{c}\text { Factor } 4 \\
\text { sexual } \\
\text { abuse }\end{array}$ \\
\hline 1 & 0.375 & & & \\
\hline 2 & 0.727 & & & \\
\hline 3 & 0.595 & & & \\
\hline 4 & 0.464 & & & \\
\hline 5 & 0.627 & & & \\
\hline 6 & 0.322 & & & \\
\hline 7 & 0.532 & & & \\
\hline 8 & 0.922 & & & \\
\hline 9 & 0.566 & & & \\
\hline 10 & 0.526 & & & \\
\hline 11 & 0.568 & & & \\
\hline 12 & & 0.868 & & \\
\hline 13 & & 0.507 & & \\
\hline 14 & & 0.890 & & \\
\hline 15 & & 0.871 & & \\
\hline 16 & & 0.828 & & \\
\hline 17 & & & 0.875 & \\
\hline 18 & & & 0.917 & \\
\hline 19 & & & 0.906 & \\
\hline 20 & & & 0.914 & \\
\hline 21 & & & 0.845 & \\
\hline 22 & & & & 0.890 \\
\hline 23 & & & & 0.914 \\
\hline 24 & & & & 0.885 \\
\hline 25 & & & & 0.800 \\
\hline 26 & & & & 0.987 \\
\hline 27 & & & & 0.767 \\
\hline
\end{tabular}

The weighted least-square with mean and variance (WLSMV) correction estimation method was used 
0.030]. Table 1 presents the factor loadings for each item.

\section{Descriptive statistics, internal consistency, and test-retest reliability}

Table 2 presents the frequency of each traumatic event, item-total correlation, Cronbach's alphas (minus item), and correlations with CTQ total. The most terrifying event question results are shown in Table 2. Participants reported that the most significant event was on the physical abuse subscale: their being 'slapped in the face.' The 'parents failed to understand your needs' item in the emotional abuse subscale, and the 'serious injury/illness of parent' in the general trauma subscale were recorded, as the second and third most terrifying events. Table 3 shows the group differences in each domain and total scores. Compared with the community sample, the clinical sample scored higher on the ETISR-SF total and its

Table 2. Frequency of endorsement and item-total correlations of items on ETISR-SF $(\mathrm{N}=304)$

\begin{tabular}{|c|c|c|c|c|c|c|c|}
\hline General trauma & \multicolumn{2}{|c|}{ Frequency } & \multicolumn{2}{|c|}{ Q1 } & $\begin{array}{l}\text { Item-total } \\
\text { correlation }\end{array}$ & $\begin{array}{c}\alpha \\
\text { (minus item) } \\
\end{array}$ & $\begin{array}{l}\text { CTQ total } \\
\text { correlation }\end{array}$ \\
\hline T1. Natural disaster & $9.2 \%$ & $(28)$ & $2.6 \%$ & $(8)$ & 0.237 & 0.870 & 0.113 \\
\hline T2. Serious accident & $13.8 \%$ & $(42)$ & $3.9 \%$ & $(12)$ & 0.494 & 0.864 & 0.183 \\
\hline T3. Serious personal injury & $21.7 \%$ & $(66)$ & $3.6 \%$ & $(11)$ & 0.444 & 0.866 & 0.198 \\
\hline T4. Serious injury/illness of parent & $20.4 \%$ & $(62)$ & $6.6 \%$ & $(20)$ & 0.355 & 0.869 & 0.190 \\
\hline T5. Separation of parents & $9.9 \%$ & $(30)$ & $3.9 \%$ & $(12)$ & 0.400 & 0.866 & 0.313 \\
\hline T6. Serious illness/injury of sibling & $10.5 \%$ & $(32)$ & $4.3 \%$ & (13) & 0.224 & 0.870 & $0.055^{*}$ \\
\hline T7. Serious injury of friend & $13.2 \%$ & $(40)$ & $2.3 \%$ & $(7)$ & 0.363 & 0.867 & 0.217 \\
\hline T8. Witnessing violence & $29.3 \%$ & $(89)$ & $5.3 \%$ & $(16)$ & 0.663 & 0.858 & 0.454 \\
\hline T9. Family mental illness & $10.2 \%$ & $(31)$ & $2.6 \%$ & $(8)$ & 0.367 & 0.867 & 0.318 \\
\hline T10. Alcoholic parents & $10.2 \%$ & $(31)$ & $2.3 \%$ & $(7)$ & 0.314 & 0.868 & 0.364 \\
\hline T11. Seeing someone murdered & $0.7 \%$ & $(2)$ & & & 0.225 & 0.869 & 0.200 \\
\hline \multicolumn{8}{|l|}{ Physical abuse } \\
\hline P1. Slapped in the face & $41.8 \%$ & $(127)$ & $7.6 \%$ & $(23)$ & 0.628 & 0.860 & 0.391 \\
\hline P2. Burned with cigarette & $14.1 \%$ & $(43)$ & $1.6 \%$ & $(5)$ & 0.393 & 0.867 & 0.225 \\
\hline P3. Punched or kicked & $34.5 \%$ & $(105)$ & $4.3 \%$ & $(14)$ & 0.651 & 0.859 & 0.409 \\
\hline P4. Hit with thrown object & $20.7 \%$ & $(63)$ & $0.7 \%$ & (2) & 0.647 & 0.859 & 0.416 \\
\hline P5. Pushed or shoved & $25.3 \%$ & $(77)$ & $0.3 \%$ & $(1)$ & 0.622 & 0.860 & 0.367 \\
\hline \multicolumn{8}{|l|}{ Emotional abuse } \\
\hline E1. Often put down or ridiculed & $17.8 \%$ & $(54)$ & $0.7 \%$ & $(2)$ & 0.637 & 0.859 & 0.441 \\
\hline $\begin{array}{l}\text { E2. Often ignored or made to feel you } \\
\text { didn't count }\end{array}$ & $30.3 \%$ & $(92)$ & $6.3 \%$ & (19) & 0.645 & 0.859 & 0.468 \\
\hline E3. Often told you are no good & $14.5 \%$ & $(44)$ & $1.3 \%$ & $(4)$ & 0.561 & 0.862 & 0.524 \\
\hline $\begin{array}{l}\text { E4. Most of the time treated in cold or } \\
\text { uncaring way }\end{array}$ & $26.3 \%$ & $(80)$ & $5.9 \%$ & (18) & 0.675 & 0.857 & 0.626 \\
\hline E5. Parents fail to understand your needs & $36.5 \%$ & $(111)$ & $6.9 \%$ & $(21)$ & 0.623 & 0.860 & 0.496 \\
\hline \multicolumn{8}{|l|}{ Sexual abuse } \\
\hline $\begin{array}{l}\text { S1. Touched in intimate parts in way that } \\
\text { was uncomfortable }\end{array}$ & $11.5 \%$ & $(35)$ & $2.3 \%$ & (7) & 0.464 & 0.864 & 0.334 \\
\hline S2. Someone rubbing genitals against you & $7.9 \%$ & $(24)$ & $0.3 \%$ & (1) & 0.449 & 0.865 & 0.304 \\
\hline S3. Forced to touch intimate parts & $3.0 \%$ & (9) & & & 0.367 & 0.867 & 0.251 \\
\hline S4. Someone had genital sex against your will & $5.6 \%$ & $(17)$ & $1.0 \%$ & (3) & 0.392 & 0.866 & 0.305 \\
\hline S5. Forced to perform oral sex & $1.6 \%$ & $(5)$ & & & 0.300 & 0.868 & 0.344 \\
\hline S6. Forced to kiss someone in sexual way & $3.3 \%$ & $(10)$ & & & 0.359 & 0.867 & 0.300 \\
\hline
\end{tabular}

Q1 is an additional question which asks to choose the most terrifying event. *the correlation coefficients are not significant at p<0.05 (twotailed). ETISR-SF: the Early Trauma Inventory Self Report-Short Form, CTQ: Childhood Trauma Questionnaire-Short Form 
subscales [for the total scale, $\mathrm{t}(304)=5.81$; for the subscales, $\mathrm{t}$ range $=2.70-5.43$, all $\mathrm{ps}<0.05$, two-tailed].

Additionally, the coefficient alpha for the entire scale was high (0.869), and the alphas of the subscales were adequate to high (0.667 to 0.832$)$. We also calculated Pearson correlations among the subscales of the ETISR-SF for each group. As presented in Table 4, the correlations between the ETISR-SF total and its subscales were moderate to high.

The ETISR-SF total and its subscales demonstrated high test-retest reliabilities over a three week period. All subscales were temporarily stable.

\section{Convergent and divergent validity}

To examine the convergent and divergent validity of the ETISR-SF, we calculated Pearson correlations with several measures, including CTQ-SF, BDI, and BAI. As shown in Table 5 , there was a strong correlation between the ETISR-SF total and the CTQ-SF total. In addition, the physical, emotional and sexual abuse subscales of the ETISR-SF showed the highest correlations with the physical, emotional, sexual abuse subscales of the CTQ-SF, respectively, indicating that the ETISR-SF total and its subscales have a good convergent validity. The correlation between ETISR-SF and the BDI was lower than the correlation between ETISR-SF and CTQ-SF, and the correlation with the BAI showed similar results, which suggest that the divergent validity of the ETISR-SF is good.

\section{DISCUSSION}

The study aimed to examine the factor structure and the psychometric properties of the Korean version of the ETISRSF in nonclinical and clinical samples. Our findings show that the Korean version of the ETISR-SF is a reliable, and is a valid

Table 3. Mean differences of ETISR-SF scores between clinical sample and non-clinical sample

\begin{tabular}{|c|c|c|c|}
\hline & $\begin{array}{c}\text { Clinical sample }(\mathrm{N}=207) \\
\text { mean }(\mathrm{SD})\end{array}$ & $\begin{array}{c}\text { Non-clinical sample }(\mathrm{N}=97) \\
\text { mean }(\mathrm{SD})\end{array}$ & $\mathrm{t}$ \\
\hline General trauma & $1.79(1.90)$ & $0.86(1.26)$ & $4.405^{*}$ \\
\hline Physical abuse & $1.63(1.64)$ & $0.80(1.17)$ & $4.437^{*}$ \\
\hline Emotional abuse & $1.59(1.77)$ & $0.54(1.04)$ & $5.431^{*}$ \\
\hline Sexual abuse & $0.42(0.99)$ & $0.13(0.49)$ & $2.703^{*}$ \\
\hline Total score & $5.43(4.87)$ & $2.33(2.82)$ & $5.813^{*}$ \\
\hline
\end{tabular}

Independent sample t-test was used. *p<0.05. ETISR-SF: the Early Trauma Inventory Self Report-Short Form

Table 4. Inter factor correlations* and test-retest reliabilities

\begin{tabular}{lcccc}
\hline & 1 & 2 & 3 & 4 \\
\hline ETISR-SF total & $0.793(0.782)$ & $0.830(0.826)$ & $0.810(0.794)$ & $0.603(0.635)$ \\
$\quad$ & & & & \\
General trauma & & & & \\
Physical abuse & $0.496(0.478)$ & 0.844 & 0.820 \\
Emotional abuse & $0.417(0.379)$ & $0.642(0.628)$ & 0.837 \\
Sexual abuse & $0.439(0.463)$ & $0.336(0.369)$ & $0.351(0.352)$ & 0.825 \\
\hline
\end{tabular}

$\mathrm{N}=304$ (whole sample). Parenthesized values are factor loadings and squared multiple correlations from the clinical sample (N=207). ${ }^{*} \mathrm{Pear}-$ son correlations were calculated, ${ }^{\dagger} \mathrm{N}=69$ (college students); the test-retest interval was three weeks. ETISR-SF: the Early Trauma Inventory Self Report-Short Form

Table 5. Convergent and divergent validity

\begin{tabular}{|c|c|c|c|c|c|c|}
\hline & \multicolumn{4}{|c|}{ CTQ-SF } & \multirow{2}{*}{$\begin{array}{c}\text { Depression } \\
\text { BDI }\end{array}$} & \multirow{2}{*}{$\frac{\text { Anxiety }}{\text { BAI }}$} \\
\hline & Total & Emotional abuse & Physical abuse & Sexual abuse & & \\
\hline ETISR-SF total & 0.691 & 0.637 & 0.629 & 0.588 & 0.424 & 0.397 \\
\hline General trauma & 0.490 & 0.406 & 0.442 & 0.483 & 0.333 & 0.304 \\
\hline Physical abuse & 0.512 & 0.503 & 0.545 & 0.393 & 0.336 & 0.327 \\
\hline Emotional abuse & 0.658 & 0.648 & 0.550 & 0.345 & 0.377 & 0.347 \\
\hline Sexual abuse & 0.459 & 0.385 & 0.379 & 0.744 & 0.229 & 0.222 \\
\hline
\end{tabular}

$\mathrm{N}=304$ (whole sample). All correlations were significant at $\mathrm{p}<0.01$ (two-tailed). Bold values indicate correlations with the corresponding subscales. Pearson correlations were calculated. ETISR-SF: the Early Trauma Inventory Self Report-Short Form, CTQ-SF: Childhood Trauma Questionnaire-Short Form, BDI: Beck Depression Inventory, BAI: Beck Anxiety Inventory 
instrument for the evaluation of childhood physical, emotional, sexual abuse, as well as general traumas.

Confirmatory factor analysis, using a whole sample, showed that our data fit well to the four-factor model originally suggested. The ETISR-SF showed high levels of internal consistency within each domain. Most items were significantly correlated with the CTQ-SF total score, and each domain of EITSR-SF showed the highest correlation with the related trauma domain of CTQ-SF, demonstrating the convergent validity of the measure. The relatively higher correlation between the physical domain and the emotional domain can be understood by the fact that these two kinds of abuse tend to occur simultaneously. ${ }^{26,27}$

The test-retest reliability of the Korean version of the ETISR-SF was slightly lower than that reported using the clinician-administered ETI in the previous study of which the test-retest reliability was $0.91 .^{1}$ In the previous study, however, the test-retest reliability of the general trauma factor $(\mathrm{r}=0.51)$ was lower than the other factors ( $\mathrm{r}$-range $=0.97-0.99$ ), showing that recalling the general trauma was relatively unstable. In our study, all subscales were temporarily stable.

The ETISR-SF has shown relatively lower correlations with the measurement of depression and anxiety than with the CTQ-SF, which suggests that the instrument measures perceived traumatic experience, rather than assessing the clinical symptoms. However the correlations with depression or anxiety were still significant, which indicates the relationship between early trauma and clinical mood symptoms in adulthood. In fact, perceived early traumas were found to be significantly associated with individual's mood symptoms in the previous study. ${ }^{5}$

It is well-known that people with many psychiatric problems, not just with PTSD, tend to report more traumatic events in their early lives. ${ }^{28}$ In the present study, the clinical sample scored significantly higher than that of the community sample, in each domain. The same aspect was presented in the original ETISR, in which the depression group scored approximately twice as much as the healthy subjects. In the original study, the score was doubled in the PTSD and borderline personality disorder groups, compared with that of the Depression group. ${ }^{1}$ When additionally analyzing the group difference between the normal control and the depression groups, total scores of the depression group were approximately twice as the scores of the normal control, which seems similar to the original group difference.

The participants in the study were drawn from a clinical setting that is specialized in depression. The data, therefore, mainly included people with depression and anxiety, and there were no PTSD patients in our study, which could be a major limitation. However, a simple comparison between the clinical and community groups does have some implication, in terms of highlighting the importance of understanding the childhood trauma. The second limitation of the study is that the test-retest reliability, which was measured only among the college students. The lack of test-retest reliability between mood episode and remission period in the clinical sample is another limitation because recalling early trauma can be affected by mood symptoms not just by memory itself. Lastly, the limitation of a cross-sectional study should be noted. The traumatic events reported by patients are perceived events, rather than facts. Future studies should include various clinical groups and assess the groups' differences. The prospective studies need to be performed to measure the actual traumatic events, and not just the perceived ones. Test-retest also needs to be measured among clinical samples, with or without mood symptoms, as to assess the effect of mood on the traumatic memory recall.

\section{Acknowledgments}

This research was supported by Basic Science Research Program through the National Research Foundation of Korea (NRF), funded by the Ministry of Education, Science and Technology (No. 2011-0013064). This study was supported by the Samsung Biomedical Research Institute grant (SBRI)(CB1-311-1).

\section{REFERENCES}

1. Bremner JD, Vermetten E, Mazure CM. Development and preliminary psychometric properties of an instrument for the measurement of childhood trauma: the Early Trauma Inventory. Depress Anxiety 2000; 12:1-12.

2. Kuo JR, Goldin PR, Werner K, Heimberg RG, Gross JJ. Childhood trauma and current psychological functioning in adults with social anxiety disorder. J Anxiety Disord 2011;25:467-473.

3. Links PS, van Reekum R. Childhood sexual abuse, parental impairment and the development of borderline personality disorder. Can J Psychiatry 1993;38:472-474.

4. Fergusson DM, Boden JM, Horwood LJ. Exposure to childhood sexual and physical abuse and adjustment in early adulthood. Child Abuse Negl 2008;32:607-619.

5. Jeon HJ, Roh MS, Kim KH, Lee JR, Lee D, Yoon SC, et al. Early trauma and lifetime suicidal behavior in a nationwide sample of Korean medical students. J Affect Disord 2009;119:210-214.

6. Nolen-Hoeksema S, Morrow J. A prospective study of depression and posttraumatic stress symptoms after a natural disaster: the 1989 Loma Prieta Earthquake. J Pers Soc Psychol 1991;61:115-121.

7. McNally RJ, Clancy SA, Schacter DL, Pitman RK. Cognitive processing of trauma cues in adults reporting repressed, recovered, or continuous memories of childhood sexual abuse. J Abnorm Psychol 2000;109:355359.

8. Berntsen D, Rubin DC. Emotionally charged autobiographical memories across the life span: the recall of happy, sad, traumatic, and involuntary memories. Psychol Aging 2002;17:636-652.

9. Bernstein DP, Ahluvalia T, Pogge D, Handelsman L. Validity of the Childhood Trauma Questionnaire in an adolescent psychiatric population. J Am Acad Child Adolesc Psychiatry 1997;36:340-348.

10. Lobbestael J, Arntz A, Harkema-Schouten P, Bernstein D. Development and psychometric evaluation of a new assessment method for childhood maltreatment experiences: the interview for traumatic events in 
childhood (ITEC). Child Abuse Negl 2009;33:505-517.

11. Bifulco A, Brown GW, Harris TO. Childhood Experience of Care and Abuse (CECA): a retrospective interview measure. J Child Psychol Psychiatry 1994;35:1419-1435.

12. Bremner JD, Bolus R, Mayer EA. Psychometric properties of the Early Trauma Inventory-Self Report. J Nerv Ment Dis 2007;195:211-218.

13. Bernstein DP, Stein JA, Newcomb MD, Walker E, Pogge D, Ahluvalia T, et al. Development and validation of a brief screening version of the Childhood Trauma Questionnaire. Child Abuse Negl 2003;27:169-190.

14. Yu JH, Park JS, Park DH, Ryu SH, Ha JH. Validation of the Korean Childhood Trauma Questionnaire: the practical use in counselling and therapeutic intervention. Korean J Health Psychol 2009;14:563-578.

15. Beck AT, Ward CH, Mendelson M, Mock J, Erbaugh J. An inventory for measuring depression. Arch Gen Psychiatry 1961;4:561-571.

16. Lee YH, Song JY. A study of the reliability and the validity of the BDI, SDS, and MMPI-D scales. Korean J Clin Psychol 1991;10:98-113.

17. Beck AT, Epstein N, Brawn G, Steer RA. An inventory for measuring clinical anxiety: psychometric properties. J Consult Clin Psychol 1998; 56:893-897.

18. Kwon SM. Differential causal roles of dysfunctional attitudes and automatic thoughts in depression. Cognit Ther Res 1992;16:309-328.

19. Sheehan DV, Lecrubier Y, Sheehan KH, Amorim P, Janavs J, Weiller E, et al. The Mini-International Neuropsychiatric Interview (M.I.N.I.): the development and validation of a structured diagnostic psychiatric interview for DSM-IV and ICD-10. J Clin Psychiatry 1998;59(Suppl 20):
22-33.

20. American Psychiatric Association. Diagnostic and statistical manual of mental disorders, 4th Edition. Washington, DC: American Psychiatric Association; 1994.

21. Tucker LR, Lewis C. A reliability coefficient for maximum likelihood factor analysis. Psychometrika 1973;38:1-10.

22. Bentler PM. Comparative fit indexes in structural models. Psychol Bull 1990;107:238-246

23. Steiger JH. Structural model evaluation and modification: an interval estimation approach. Multivariate Behav Res 1990;25:173-180.

24. Hu LT, Bentler PM. Cutoff criteria for fit indexes in covariance structure analysis: conventional criteria versus new alternatives. Struct Equ Modeling 1999;6:1-55.

25. Browne MW, Cudeck R. Alternative ways of assessing model fit. Sociol Methods Res 1992;21:230-258.

26. Briere J, Runtz M. Multivariate correlates of childhood psychological and physical maltreatment among university women. Child Abuse Negl 1988;12:331-341.

27. Lee JS, Kim EK. Effect of juveniles' experience of being abused on their shame-proneness and parentification. Korean J Couns Psychother 2007;19:339-356.

28. Coverdale JH, Turbott SH. Sexual and physical abuse of chronically ill psychiatric outpatients compared with a matched sample of medical outpatients. J Nerv Ment Dis 2000;188:440-445. 